\section{The optical counterpart to the $\gamma$-ray burst GRB970508}

\section{S. G. Djorgovski ${ }^{\star}$, M. R. Metzger ${ }^{\star}$, S. R. Kulkarni ${ }^{\star}$, S. C. Odewahn*, R. R. Gal ${ }^{\star}$, M. A. Pahre ${ }^{\star}$, D. A. Frail $\dagger$, M. Feroci $\ddagger$, E. Costa $\ddagger$ \& E. Palazzi $\S$}

* Palomar Observatory, California Institute of Technology, Pasadena, California 91125, USA

$\dagger$ National Radio Astronomy Observatory, Socorro, New Mexico 87801, USA $\ddagger$ Istituto di Astrofisica Spaziale CNR, 00044 Frascati, Italy

$\$$ Istituto Technologie e Studio Radiazioni Extraterrestri CNR, 40129 Bologna, Italy

Understanding the nature of the $\gamma$-ray burst phenomenon is one of the outstanding problems of modern astrophysics. The identification of counterparts at optical wavelengths is considered a crucial factor for determining the origin of these events. Here we report the detection and temporal properties of a variable optical source, which has been identified ${ }^{1,2}$ as the counterpart of the X-ray transient associated with the $\gamma$-ray burst GRB970508 (ref. 3). The initial optical images were obtained only 5.8 hours after the initial $\gamma$-ray burst, after which the optical source was observed to brighten over the next two days before declining in luminosity with a $t^{-1}$ power law. The decline in brightness follows a form predicted by many relativistic fireball models ${ }^{4-7}$ for $\gamma$-ray bursts, although the initial rise does not appear to be compatible with the simplest of these models. The observed fluence of the source at visible wavelengths over the period spanned by our observations is $\geqslant 4.6 \times 10^{-8} \mathrm{erg} \mathrm{cm}^{-2}$, about $3 \%$ of the fluence of the $\gamma$-ray burst itself.

Since their discovery nearly three decades $\operatorname{ago}^{8}$, the understanding of $\gamma$-ray bursts (GRBs) has presented a major challenge for astrophysicists, generating an extensive literature ${ }^{9-11}$. Until recently, progress has been stymied by poor localization at $\gamma$-ray wavelengths. It has long been recognized that the key to understanding the nature and origin of GRBs is their detection in some other wavelength regime, preferably in the visible band which has the advantage of spectroscopy, whereby distances could be determined.

The field has been revolutionized with the launch of the BeppoSAX satellite ${ }^{12}$, which can provide positions accurate to a few arcmin within hours, and accurate to 1 arcmin (or better) within a fraction of a day. This has already resulted in a major advance: the discovery that GRBs are followed by bright X-ray sources which decay on timescales of the order of a few days (refs 13-15). BeppoSAX can localize these X-ray sources considerably more precisely than their $\gamma$-ray positions, to a 45 -arcsec radius, or better. This enables rapid follow-up observations at optical and other wavelengths. Another significant new development has been the detection of a fading optical counterpart ${ }^{16}$ within the precise localization of the fading X-ray counterpart of GRB970228. No fading optical counterpart was $\operatorname{seen}^{17}$ in the case of GRB970402, perhaps due to its proximity to the Galactic plane.

Here we report on the optical detection and follow-up observations of the fading X-ray source associated with GRB970508 ${ }^{3}$. The optical counterpart was first reported by Bond ${ }^{1}$, and was independently discovered at about the same time in our data ${ }^{2}$. Our observations were conducted on the Palomar 200-inch Hale telescope (hereafter P200) and the Palomar 60-inch telescope (hereafter $\mathrm{P} 60)$. On the P200 we used a prime-focus charge-coupled device (CCD) imager, which has a field of view of 13.6 arcmin with 0.40 arcsec pixels. On the P60 we used a CCD camera attached to the Cassegrain focus, with a field of 12.8 arcmin and 0.372 -arcsec pixels. All observations were in the Gunn gri photometric system.

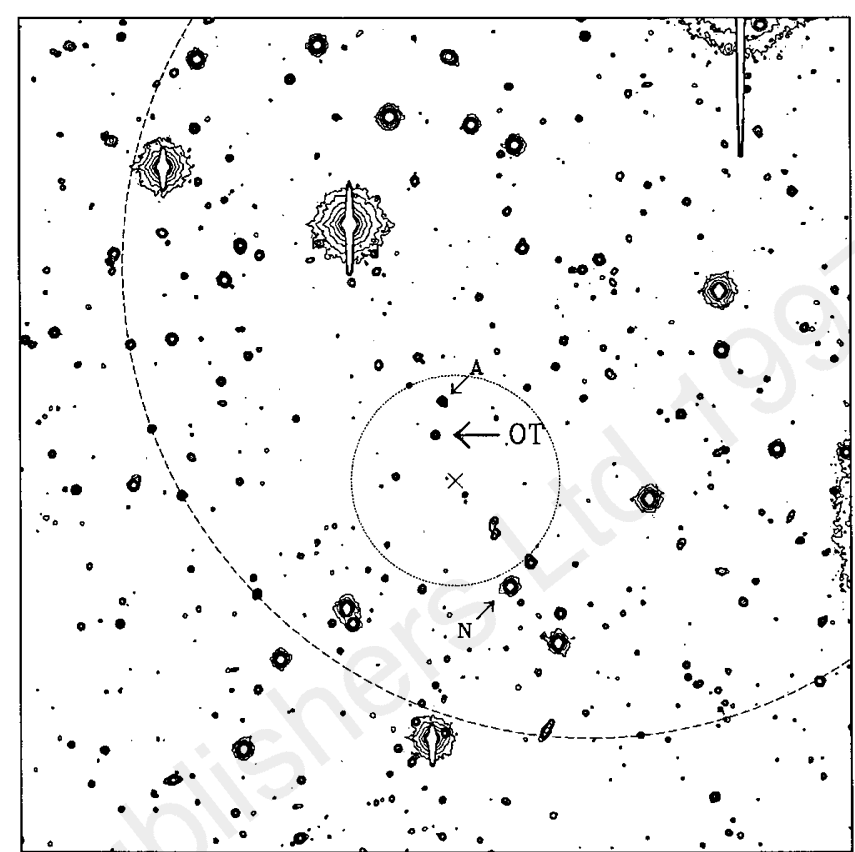

Figure 1 Finding chart for the field of GRB970508, from an r-band image obtained at Palomar on 1997 May 10 ut. The field shown is 320-arcsec square, with north at the top and east to the left. The proposed optical counterpart of the burst (OT) is indicated (see text). We also indicate two nearby offset stars, labelled 'A' (2.7 arcsec west and 13.0 arcsec north of the OT, $r=19.85 \mathrm{mag}$ ) and ' $\mathrm{N}$ ' (28.9 arcsec west and 58.3 arcsec south of the OT, $r=17.4 \mathrm{mag}$ ). The dotted circle centred on the cross, with a 40-arcsec radius, indicates the error circle of the $\mathrm{X}$-ray transient ${ }^{15}$. The dashed circle, with a radius of $3 \mathrm{arcmin}$, indicates the final error circle of the GRB ${ }^{18}$.

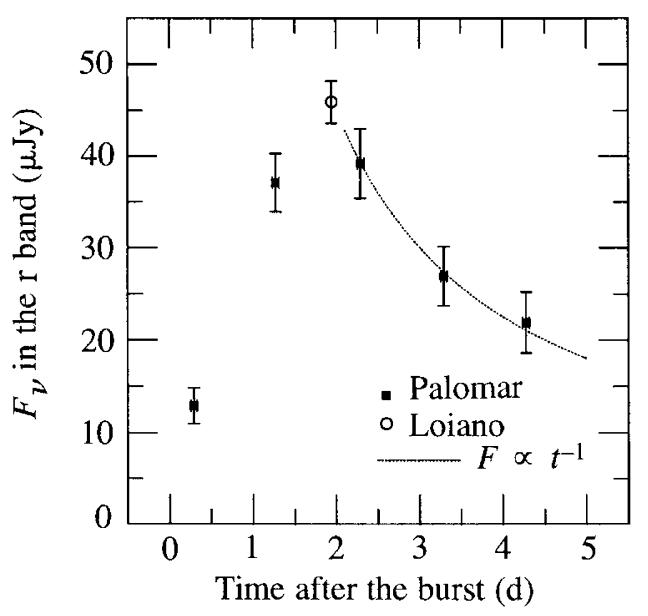

Figure 2 Optical light curve in the $r$ band, as a function of time starting at May 8.904 ut, from the Palomar data listed in Table 1 (filled squares). The data have been corrected for the Galactic extinction and converted to specific fluxes as described in the text. These numbers supersede our preliminary measurements ${ }^{28}$. We also include a Loiano Observatory measurement ${ }^{29}$ of $R_{C}=19.78 \pm 0.05 \mathrm{mag}$ (in the Kron-Cousins system) at the mean epoch of May 10.85 , close to the peak of the light curve (empty circle). After correcting for the extinction and using the $R_{C}$ zero-point calibration ${ }^{30}$, we derive $F_{\nu}=45.9 \mu \mathrm{Jy}$ at the effective wavelength $\lambda_{\text {eff }} \approx 641 \mathrm{~nm}$, close to the centre of the $r$ band. We do not use other magnitudes reported in the IAU Circulars so far, owing to the lack of information about the photometric systems used, photometric conditions, and so on. The decline in brightness seen after 10 May is consistent with a power-law $F_{v} \propto t^{-1}$, with time counting from the GRB event, shown as the dotted line drawn through our 11 May data point. 


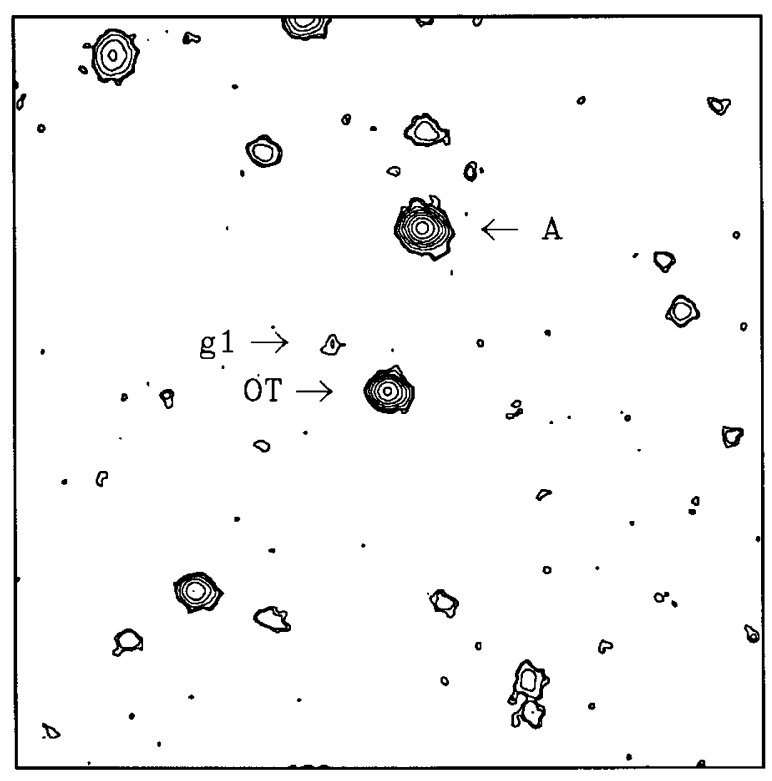

Figure 3 A section of a deep r-band image (average of our P200 observations on 9 and 10 May), centred on the OT. The field shown is 1 -arcmin square, with north at the top, and east to the left. A faint, $\sim 24.5$ mag galaxy (' $g 1$ ') is seen 4.3 arcsec east and 3.5 arcsec north of the OT. It may be associated with the host galaxy of the OT itself, or with one of the absorption line systems seen in its spectrum ${ }^{25,27}$. The feature labelled 'A' is the offset star marked in Fig. 1.

The first set of observations were conducted on P200 starting only 5.8 hours after the burst. Multiple exposures in the Gunn r band were obtained in several pointings to cover the large 10 -arcmin BeppoSAX Wide Field Camera error circle $^{3}$. Typical limiting magnitudes were $r \approx 23.5 \mathrm{mag}$ for the individual 5-minute exposures, and $r \approx 24.5 \mathrm{mag}$ for the average stack of them. No source brighter than $r \approx 22$ mag was seen to vary by more than $20 \%$ over the baselines of about 1-2 hours in these data. On the next night, 1997 May 10 uT, images were obtained but this time centred on the revised GRB position ${ }^{18}$, with an error radius of $3 \mathrm{arcmin}$. Some images in the Gunn $\mathrm{g}$ and $\mathrm{i}$ bands were also obtained on this night.

On the same night, 10 May, gri observations were also made at the P60 and calibrated using standard stars ${ }^{19}$. These data were used to establish a grid of secondary photometric stars in the field. The calibration observations were obtained under photometric conditions. The observations of this night define the photometric zeropoints, which we estimate to be good to $\Delta g \leqslant 0.08 \mathrm{mag}$, $\Delta r \leqslant 0.06 \mathrm{mag}$, and $\Delta i \leqslant 0.08 \mathrm{mag}$ (conservative errors). All the magnitudes are referred to this grid of secondary stars.

Subsequent observations were obtained at the P60 on 1997 May 11.149 to 11.238 UT, and May 12.156 to 12.235 UT, and at the P200 on 1997 May 13.164 to 13.194 UT. Some of these observations were obtained in non-photometric conditions, but because we use relative photometry from a grid of secondary photometric stars in the field, corrected magnitudes can still be obtained. From the scatter of magnitude differences for different nights, we estimate that these offsets are determined to $0.02-0.03 \mathrm{mag}$ accuracy.

The comparison of P200 data between 9 and 10 May indicates several possible variable sources over the entire 3-arcmin-radius GRB error circle. Variable candidates were identified in an automated fashion using a combination of the photometry program DoPHOT $^{20}$ and an object cataloguing package ${ }^{21}$. However, only one

\begin{tabular}{|c|c|c|c|c|}
\hline $\begin{array}{l}\text { Date } \\
\text { (UT) }\end{array}$ & Telescope & Band & Magnitude & $\begin{array}{c}F_{\nu} \\
(\mu J y)\end{array}$ \\
\hline May 9.195 & P200 & $r$ & $21.35 \pm 0.15$ & 12.9 \\
\hline May 10.199 & P200 & $g$ & $20.25 \pm 0.10$ & 29.5 \\
\hline May 10.178 & P200 & r & $20.20 \pm 0.09$ & 37.1 \\
\hline May 10.238 & P200 & i & $20.22 \pm 0.10$ & 38.8 \\
\hline May 11.198 & P60 & r & $20.14 \pm 0.10$ & 39.2 \\
\hline May 12.195 & P60 & $r$ & $20.55 \pm 0.12$ & 26.9 \\
\hline May 13.179 & P200 & $r$ & $20.77 \pm 0.15$ & 21.9 \\
\hline
\end{tabular}

of them - the highest-amplitude object-is within the X-ray transient source error circle $^{15}$. We measure for its position in the US Naval Observatory's A1.0 reference system: right ascension (RA) 06 h 53 min $49.43 \mathrm{~s}$, declination (dec.) +79 16' 19.6" (J2000) with uncertainties of $\sim 1$ arcsec. The same optical transient (hereafter OT) was reported by Bond ${ }^{1}$. We measure brightening of $1.15 \pm 0.10 \mathrm{mag}$, from $r=21.35 \pm 0.15 \mathrm{mag}$ on May $9.195 \mathrm{UT}$, to $r=20.20 \pm 0.09 \mathrm{mag}$ on May 10.178 uT (mean epochs). Given that the source is within the 45 -arcsec (radius) error circle of the X-ray transient, and given its peculiar light curve, we conclude that this is the probably optical counterpart of the X-ray transient, which in turn is most likely to be associated with GRB970508. A finding chart for the object is shown in Fig. 1.

From the Infra-Red Astronomy Satellite (IRAS) $100-\mu \mathrm{m}$ cirrus flux in this direction ${ }^{22}$ we derive for the Galactic extinction $A_{B}=0.11$ mag. Using the interpolation of the extinction curve ${ }^{23}$, we derive extinction in the gri bands of $A_{g}=0.09 \mathrm{mag}$, $A_{r}=0.07 \mathrm{mag}$ and $A_{i}=0.05 \mathrm{mag}$. These corrections are applied to all of our measurements listed below.

Table 1 summarizes the photometric results on the OT, after applying the extinction corrections above. Using the flux-standard stars ${ }^{24}$, we can convert these magnitudes to specific fluxes; we assume that $r=0$ mag corresponds to the specific flux $F_{\nu}=4,460 \mathrm{Jy}$. The effective wavelength of the Gunn $\mathrm{r}$ band is $\lambda_{\text {eff,r }} \approx 665 \mathrm{~nm}$. The light curve is shown in Fig. 2 .

On May 10.178, the extinction-corrected colours of the source were $(g-r)=+0.07 \mathrm{mag}$ and $(r-i)=-0.09 \mathrm{mag}$. To bring all measurements to a common epoch, we assumed a brightening rate of $0.05 \mathrm{mag} \mathrm{h}^{-1}$ in all three bands. Assuming that $g=0 \mathrm{mag}$ corresponds to $F_{\nu}=3,720 \mathrm{Jy}$ at $\lambda_{\text {eff, }} \approx 520 \mathrm{~nm}$, and $i=0 \mathrm{mag}$ to $F_{\nu}=4,750 \mathrm{Jy}$ at $\lambda_{\text {eff, }} \approx 790 \mathrm{~nm}$, we can fit the gri fluxes with a power-law continuum of the form $F_{\nu} \propto \nu^{\alpha}$. We get $\alpha=$ $-0.65 \pm 0.3$, in excellent agreement with the slope estimated from the nearly simultaneous spectroscopy. ${ }^{25}$

The light curve shows an initial rise and then a decline (starting on 11 May) consistent with a $t^{-1}$ (or, more precisely, $t^{-0.9 \pm 0.1}$ ) dependence, where $t$ is the time since the burst. This is indeed predicted by the fireball model ${ }^{4-7}$. This is a generic model in which a large amount of energy is rapidly released in a small volume. Initially, the deposited energy is optically thick to pair creation $\left(\gamma \gamma \rightarrow e^{+} e^{-}\right)$, and consequently radiation pressure drives a rapid expansion in a fireball stage. The expansion cools the electrons and all the energy appears as kinetic energy of the baryons in a bulk outflow phase. When this shell meets the interstellar medium, the resulting shock produces broad-band emission. In this model, the initial $\gamma$-ray burst is expected to be followed by X-ray, optical and radio outbursts ${ }^{7,26,31}$, as indeed observed ${ }^{32}$ for GRB970508.

However, we also see a relatively slow rise from 9 to 10 May. If the rise was exponential between these two points, the $e$-folding time (that is, the time in which the flux would increase by a factor of $e=2.718 \ldots$ ) would be $\sim 22$ hours in the observed reference frame. This is not predicted in the simplest fireball model, where the onset of the optical emission should be very $\operatorname{rapid}^{7}$. But, inverse Compton 
cooling of relativistic electrons in the early stages of expansion could account for such a behaviour ${ }^{33}$.

We integrate the r-band light curve over the time interval spanned by our observations to obtain a total fluence. We interpolate linearly in time between the measured magnitudes, and integrate the product $\nu F_{\nu}$ where $\nu$ is the effective frequency in the $\mathrm{r}$ band, between the first and the last of our observations to obtain the total fluence in the visible (observed red) light $I_{\mathrm{r}}>4.6 \times 10^{-8} \mathrm{erg} \mathrm{cm}^{-2}$. This is a lower limit, as we do not include the unknown portions of the light curve outside our measurements, and we do not sample well the portion near maximum light. For comparison, the $\gamma$-ray burst had a fluence of $I_{\gamma} \approx(1.8 \pm 0.3) \times 10^{-6} \mathrm{erg} \mathrm{cm}^{-2}$, derived from BeppoSAX observations in the energy band $40-700 \mathrm{keV}$. We thus conclude that at least $3 \%$ of the total GRB energy is emitted as visible light.

Figure 3 shows a stack of all of our P200 images from 9 and 10 May, centred on the source. A faint, blue galaxy (labelled 'g1') with $g \approx 24.5 \pm 0.4 \mathrm{mag}$ and $r \approx 24.8 \pm 0.4 \mathrm{mag}$ is clearly detected 4.3 arcsec east and 3.5 arcsec north of the OT. The two may be physically associated, or the galaxy could be responsible for some of the absorption lines seen in the source spectrum reported by Metzger et al. ${ }^{25,27}$. Its magnitude is typical of the general field galaxy population at $z \approx 0.8$, and its blue colour is suggestive of active star formation.

Received 21 May; accepted 28 May 1997.

. Bond, H. E. IAU Circ. No. 6654 (1997).

2. Djorgovski, S. et al. IAU Circ. No. 6655 (1997).

Costa, E. et al. IAU Circ. No. 6649 (1997).

4. Paczyns̀ki, B. Gamma-ray bursters at cosmological distances. Astrophys. J. 308, L43-L46 (1986).

. Goodman, J. Are gamma-ray bursts optically thick? Astrophys. J. 308, L47-L50 (1986).

6. Mészáros, P. \& Rees, M. J. Optical and long-wavelength afterglow from gamma-ray bursts. Astrophys. J. 476, 232-237 (1997)

Wijers, R. A. M. J., Rees, M. J. \& Mészáros, P. Shocked by GRB 970228: the afterglow of a cosmological fireball. Mon. Not. R. Astron. Soc. (in the press); preprint http://xxx.lanl.gov/astro-ph/9704153.

8. Klebasadel, R. W., Strong, I. B. \& Olson, R. A. Observations of gamma-ray bursts of cosmic origin. Astrophys. J. 182, L85-L88 (1973).

9. Fishman, G. J. \& Meegan, C. A. Gamma-ray bursts. Annu. Rev. Astron. Astrophys. 33, 415-458 (1995).

10. Lamb, D. Q. The distance scale to gamma-ray bursts. Publ. Astron. Soc. Pacif. 107, 1152-1166 (1995).

11. Paczyǹski, B. How far away are gamma-ray bursters? Publ. Astron. Soc. Pacif. 107, 1167-1175 (1995).

12. Boella, G. et al. BeppoSAX, the wide band mission for X-ray astronomy. Astron. Astrophys. Suppl. Ser. 122, 299-399 (1997)

13. Costa, E. et al. IAU Circ. No. 6572; (1997); Discovery of an X-ray afterglow associated with the $\gamma$-ray burst of 28 February 1997. Nature 387, 783-785 (1997).

14. Piro, L. et al. IAU Circ. No. 6617 (1997).

15. Piro, L. et al. IAU Circ. No. 6656 (1997).

16. van Paradijs, J. et al. Transient optical emission from the error box of the gamma-ray burst of 28 February 1997. Nature 386, 686-689 (1997).

17. Harrison, T. et al. LAU Circ. No. 6632 (1997).

18. Heise, J. et al. IAU Circ. No. 6654 (1997).

19. Kent, S. M. Photometry of stars in the uvgr system. Publ. Astron. Soc. Pacif. 97, 165-174 (1985).

20. Schechter, P. L., Mateo, M. \& Saha, A. DoPHOT, a CCD photometry program-description and tests. Publ. Astron. Soc. Pacif. 105, 1342-1353 (1993).

21. Metzger, M. R. Constraints on the shape and size of the Galactic gravitational potential. Thesis, Massachusetts Inst. Technol. (1994)

22. Laureijs, R. J., Helou, G. \& Clark, F. O. The First Symp. on the Infrared Cirrus and Diffuse Interstella Clouds (eds Cutri, R. \& Latter, W.) 133-136 (Conf. Ser. 58, Astron. Soc. Pacif., Provo, UT, 1994).

23. Seaton, M. J. Interstellar extinction in the UV. Mon. Not. R. Astron. Soc. 187, 73P-76P (1979).

24. Oke, J. B. \& Gunn, J. E. Secondary stars for absolute spectrophotometry. Astrophys. J. 266, 713-717 (1983).

25. Metzger, M. R. et al. Spectral constraints on the redshift of the optical counterpart to the $\gamma$-ray burst of 8 May 1997. Nature 387, 878-880 (1997).

26. Mészáros, P. \& Rees, M. J. Gamma-ray burst models. Astrophys. J. 397, 570-575 (1992).

27. Metzger, M. R. et al. IAU Circ. No. 6655 (1997).

28. Djorgovski, S. et al. IAU Circ. No. 6658 (1997).

29. Mignoli, M. et al. IAU Circ. No. 6661 (1997).

30. Bessel, M. UBVRI passbands. Publ. Astron. Soc. Pacif. 102, 1181-1199 (1990).

31. Paczyński, B. \& Rhoads, J. E. Radio transients from gamma-ray bursters. Astrophys. J. 418, L5-L8 (1993).

32. Frail, D. A. \& Kulkarni, S. R. IAU Circ. No. 6662 (1997)

33. Waxman, E. GRB after-glow: supporting the cosmological fireball model, constraining parameters, and making predictions. Astrophys. J. (in the press); preprint http://xxx.lanl.gov/astro-ph/9704116 (1997)

Acknowledgements. We thank the staff of Palomar Observatory for their assistance during our observing runs, and the entire BeppoSAX team for their efforts in providing accurate burst positions as rapidly as possible. This work was supported in part by the NSF, NASA, the Bressler Foundation, the Norri Foundation and Caltech.

Correspondence should be addressed to S.G.D. (e-mail: george@oracle.caltech.edu).
Spectral constraints on the redshift of the optical counterpart to the $\gamma$-ray burst of 8 May 1997

\author{
M. R. Metzger ${ }^{\star}$, S. G. Djorgovski*, S. R. Kulkarni*, \\ C. C. Steidel ${ }^{\star}$, K. L. Adelberger ${ }^{\star}$, D. A. Frail $\dagger$, E. Costa \\ \& F. Frontera $\$$
}

* Palomar Observatory, 105-24, California Institute of Technology, Pasadena, California 91125, USA

$\dagger$ National Radio Astronomy Observatory, Socorro, New Mexico 87801, USA

$¥$ Istituto di Astrofisica Spaziale CNR, 00044 Frascati, Italy

$\$$ Dipartimento di Fisica, Universita' di Ferrara and Istituto TESRE-CNR,

40129 Bologna, Italy

Brief, intense bursts of $\gamma$-rays occur approximately daily from random directions in space, but their origin has remained unknown since their initial detection almost 25 years ago ${ }^{1}$. Arguments based on their observed isotropy and apparent brightness distribution ${ }^{2}$ are not sufficient to constrain the location of the bursts to a local $^{3}$ or cosmological origin ${ }^{4}$. The recent detection of a counterpart to a $\gamma$-ray burst at other wavelengths $s^{5,6}$ has therefore raised the hope that the sources of these energetic events might soon be revealed. Here we report spectroscopic observations of the possible optical counterpart ${ }^{7,8}$ to the $\gamma$-ray burst GRB970508. The spectrum is mostly featureless, except for a few prominent absorption lines which we attribute to the presence of an absorption system along the line of sight at redshift $z=0.835$. Coupled with the absence of Lyman- $\alpha$ forest features in the spectra, our results imply that the optical transient lies at $0.835 \leqslant z \leqslant 2.3$. If the optical transient is indeed the counterpart of GRB970508, our results provide the first direct limits on the distance to a $\gamma$-ray burst, confirming that at least some of these events lie at cosmological distances, and are thus highly energetic.

On 8 May 1997 UT, a moderate-fluence classical $\gamma$-ray burst (GRB970508) was detected by instruments aboard the ItalianDutch satellite BeppoSAX ${ }^{9}$; the burst was localized initially to an error region of 5 -arcmin radius ${ }^{10}$ and later to a region of 3 -arcmin radius $^{11}$. A potential optical counterpart was identified within two days $^{7,8}$, which we refer to as OT J065349+79163 (here OT stands for optical transient). Interest in this object was heightened when the appearance of a bright X-ray source was reported ${ }^{12}$ that was not seen in a previous X-ray all-sky survey, and included OT J065349+79163 in the 45 -arcsec-radius error circle. The presence of a new and bright $\mathrm{X}$-ray source in a $\gamma$-ray burst (GRB) error circle has been seen in the recent three GRBs observed by BeppoSAX. In the first such case, a decaying optical source ${ }^{5}$ was also seen and these authors suggested that such fading (X-ray and optical) sources are the afterglow of $\gamma$ ray bursts. It has been suggested ${ }^{13}$ that because OT J065349+79163 exhibits unusual variability at optical and X-ray wavelengths, it represents a similar optical afterglow to GRB970508.

We obtained spectra of OT J065349+79163 with the Keck II 10-m telescope on 11 May 1997 UT, using the Low Resolution Imaging Spectrograph $^{14}$ (LRIS) with a $2,048 \times 2,048$ pixel CCD (chargecoupled device). Starting at 05:44 UTC, a sequence of three 10minute spectra were obtained with a 1.0-arcsecond-wide slit oriented along the direction of the atmospheric dispersion. The spectrograph was configured with a 300 lines $\mathrm{mm}^{-1}$ grating blazed at $5,000 \AA$, covering the region $3,850-8,550 \AA$. We obtained two further spectra of 10 minutes each on 12 May 1997 UT. Calibration $\mathrm{Hg}-\mathrm{Kr}-\mathrm{Ar}$ and flat lamp spectra were taken at the end of each exposure sequence. Owing to the extremely low elevation angle of 